\title{
Hyper-Fine Features of the Dust Distribution in the Central Region of the Seyfert Galaxy NGC 4151
}

\author{
Hiroshi Ohtani, Takashi Hattori \\ Department of Astronomy, Kyoto University, Kyoto, Japan \\ Satoshi Miyazaki, Masanori Iye \\ National Astronomical Observatory, Mitaka, Japan \\ Yutaka Komiyama \\ Department of Astronomy, University of Tokyo, Tokyo, Japan \\ Toshiyuki Sasaki, George Kosugi and Subaru team \\ Subaru Telescope, Hilo, Hawaii, USA
}

\begin{abstract}
.
Fine features of the dust distribution in the central region of NGC 4151 have been studied from high resolution images obtained by the Subaru Telescope. The two dust lanes lying along a circum-nuclear ellipse of 40 " $\times 20$ " have been recognized to consist of a number of fine filaments and knots. In the inner region, there has been found dust distributed in a bipolar cone region extending to 5 " from the nucleus. This cone is exactly complementary to the well-known bipolar ionized gaseous region. A dense knot is seen at 2" SE from the nucleus, but no torus-like feature is recognized. The masses of neutral gas in the outer lanes and in the inner region are estimated as $10^{6} M_{\odot}$ and $10^{5} M_{\odot}$, respectively. These observational results are compared with results of numerical simulations of gas dynamics.
\end{abstract}

\section{Introduction}

An important implication of the current idea that an active galactic nucleus harbours a giant black hole is the presence of some yet unidentified mechanism for fueling gas efficiently to the accretion disk around the black hole. The fueling process is occasionally considered in connection with the dusty torus which is assumed to explain the observed properties of type 1 and 2 of Seyfert nuclei in the unified scheme.

Here, we present a study of dust distribution of the nuclear region of NGC4151 based on high $\mathrm{S} / \mathrm{N}$ images of $\mathrm{V}$ and I bands with a resolution as high as 0.5 " obtained by the Subaru telescope. As the distance to NGC4151 is $13.3 \mathrm{Mpc}, 1$ " corresponds to $64 \mathrm{pc}$. 


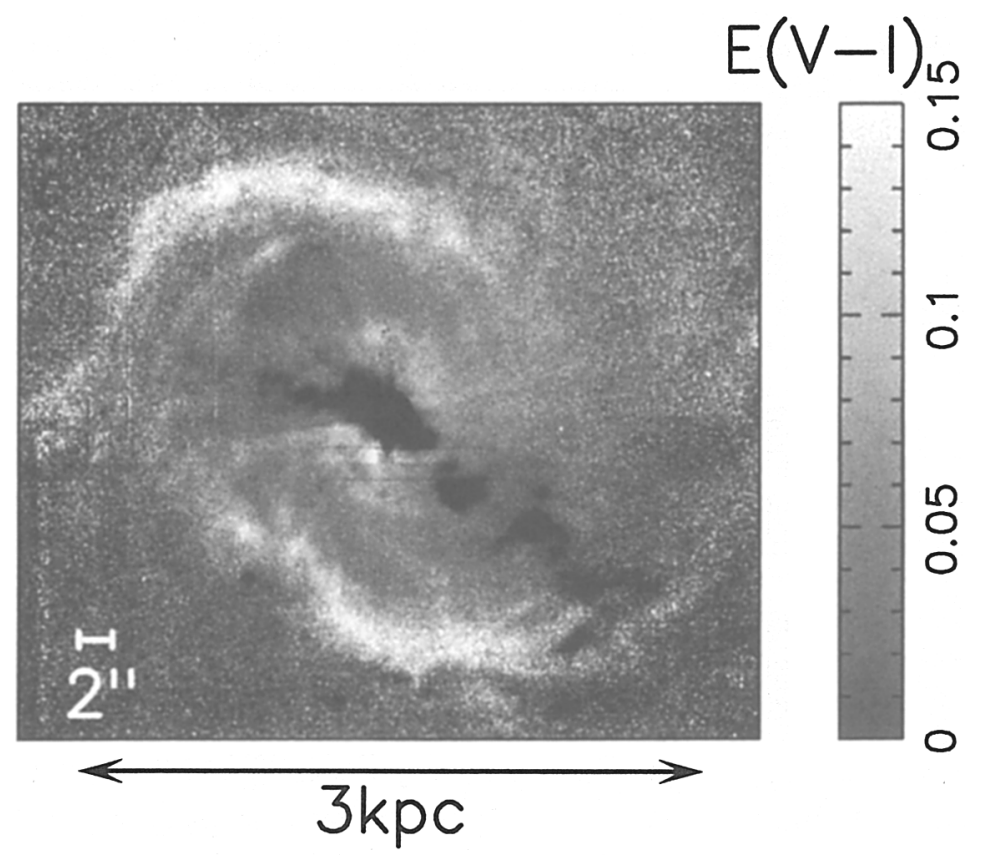

Figure 1. $\quad \mathrm{E}(\mathrm{V}-\mathrm{I})$ map of the central region of NGC4151

\section{Dust gas distribution}

From these images we constructed a V-I color image. Since flux calibration was not made, the zero point of the color is not determined. As the intrinsic V-I of the stellar component in the bulge region of NGC4151 is nearly constant from color maps of Vila-Vilaro et al. (1995), we made an image of the distribution of difference of the color from that of the stellar background. Then, the positive difference is interpreted as color excess $\mathrm{E}(\mathrm{V}-\mathrm{I})$ due to reddening by dust. The result is shown in Figure 1 as a grey scale image, where the lighter regions than the background correspond to more reddening. The color excess was converted into visual extinction using the standard reddening law.

In Figure 1, the two dust lanes first found by Vila-Vilaro et al. (1995) are seen very clearly. These are well fitted to two arcs of an ellipse of semimajor axis 20 " $\times 10$ " with the center at the nucleus. Many fine filamentary and knotty structures are disclosed by the present image. The visual extinction of the densest knot is found to be about $A_{V}=0.4 \mathrm{mag}$ both in the northern and southern arc. From the shape of the dust lanes and their HI velocities measured by Asif et al. (1998), Ulrich (2000) has suggested that the ellipse corresponds to the $x_{2}$ orbits along which gas supplied from the bar region is moving.

In addition to the dust lanes, it has been found that there is significant dust extinction in the inner region encircled by the two dust lanes. The dust is dominantly in a bipolar cone region extending to 5" from the nucleus. The axis of the bipolar cone is oriented approximately in NW and SE, and the opening 
angle is about 110 degree for both directions. The distribution of the inner dust is rather diffuse compared with the outer dust lane, but shows fine structures; knotty structures and also weak spiral-like ones can be identified. The small dust knot noticed by Asif et al.(1998) near the nucleus is found to be the densest knotty part $\left(A_{V}=0.4 \mathrm{mag}\right)$ which is located at $2 " \mathrm{SE}$ from the nucleus. No torus-like structure is seen.

\section{Circumnuclear gaseous system}

In Figure 1, ionized gaseous regions are also distinguished as regions with negative reddening, i.e., as the regions which are darker than the background. This is due to contamination of $\mathrm{V}$ band by the strong [OIII] emission lines from the ionized gas. It is quite interesting that dusty bipolar cone is exactly complementary to the well known bipolar cone of ionized gas which is seen as the darker part acrossing the nucleus from NE to SW. It is further interesting that the features of the internal structures of the distribution of the ionized gas are quite similar to that of the dusty gas. The diffuse [OIII] emission radially decreases similarly to the dust distribution. The bright [OIII] knots are similar to the dense neutral knots. The arc-like structure extending azimuthally at the south west end of the ionized cone lie in contact with the inside of the dust lane there. This suggests the inner side of the dust lane is ionized by the nuclear radiation. It is known that the motion of the ionized gas is mostly a circular rotation in the galactic plane (e.g., Winge et al. 1999). Therefore, it is natural to consider that there is a circumnuclear gaseous disk in the galactic plane and that a bipolar sector exposed to ionizing radiation from the nucleus is seen as the bipolar ionized cone.

The extinction was converted into molecular hydrogen column density by using the ratio $1.25 \times 10^{21} \mathrm{~cm}^{-2} \mathrm{mag}^{-1}$ for galactic dark clouds (Dickman 1978), and the masses of the various regions were estimated. The results are shown in Figure 2. The masses of the northern and the southern arcs are $3.5 \times 10^{6} M_{\odot}$ and $1.5 \times 10^{6} M_{\odot}$, while the mass of the bipolar fan (a better name than cone) is $4.7 \times 10^{5} M_{\odot}$ and $1.5 \times 10^{5} M_{\odot}$ for the $\mathrm{NW}$ and the $\mathrm{SE}$, respectively. The mass of the densest knots close to the nucleus is estimated as $3 \times 10^{4} M_{\odot}$. This value is compared with those of masses of the ionized gaseous knots derived from $\mathrm{H} \alpha$ emission by Yoshida and Ohtani(1993). This supports the suggestion stated above that the ionized gas and the neutral gas are originally the same gaseous constituent distributed in the galactic plane in the nuclear region.

\section{Relation to fueling mechanism}

One may expect that the neutral gas in the newly found bipolar fan region might be gas being fed into the Seyfert nucleus. In this context, it should be noticed that the overall feature of the distribution of the neutral and ionized gas are reproduced quite well by a gas dynamical simulation by Fukuda, Wada and Habe (2000), who took into account the effect of self-gravity of gas in a model of a weakly barred galaxy harbouring a super-massive black hole at the nucleus. They set the values of the dynamical parameters of their model galaxy as those not so far different from the observed ones in NGC4151, and obtained a mass 


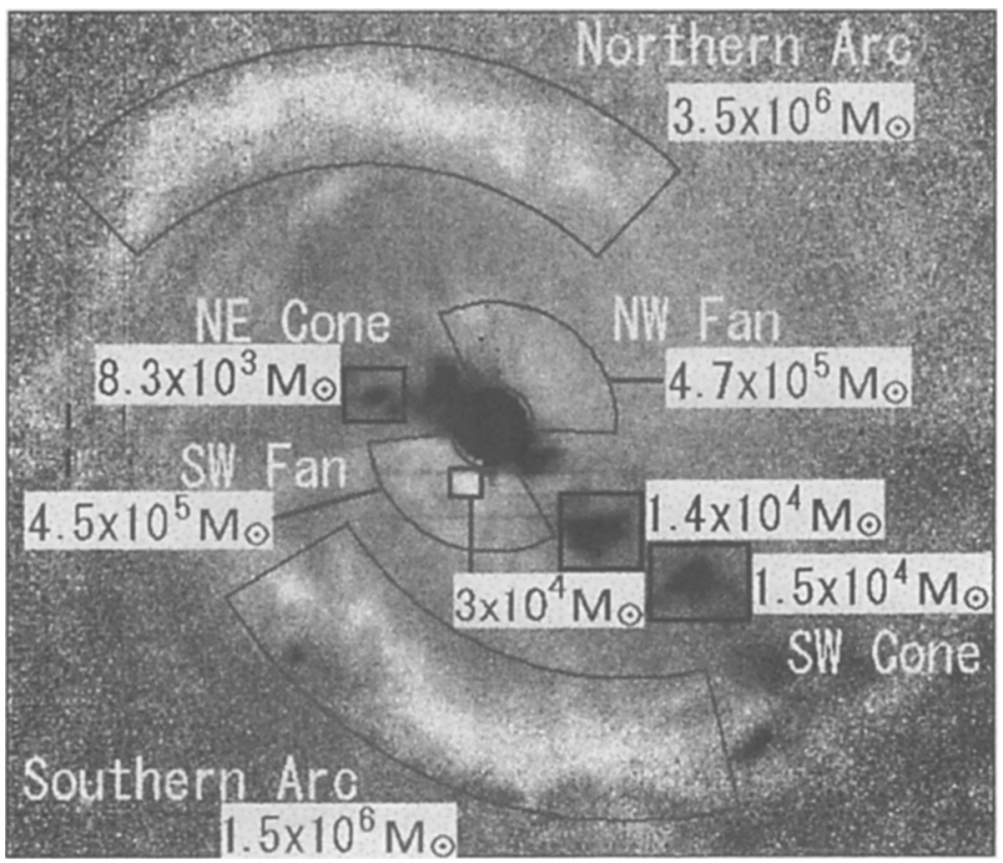

Figure 2. Masses of neutral gas of various regions derived from extinction. Values for the ionized gaseous knots are from $\mathrm{H} \alpha$ emission.

accretion rate into a region of $50 \mathrm{pc}$ from the galactic center as $0.1 M_{\odot} / y r$ for $3.5 \times 10^{7}$ years.

In order to examine the relation of the neutral gas in the newly found bipolar fan region to the gas fueling mechanism, kinematic information of this region is essential.

\section{References}

Asif, M. W., Mundell, C. G., Pedler, A., Unger, S. W., Robinson, A., Vila-Vilaro, B. and Lewis, J. R., 1998, A\&A, 333, 466

Dickman, R. L., 1978, AJ, 83, 363

Fukuda, H., Habe, A. and Wada, K., 2000, ApJ, 529, 109

Ulrich, M-E., 2000, Astronomy and Astrophysics Review, 10, 135

Vila-Vilaro, B., Robinson, A., Perez, E., Axon, E. J., Baum, S. A. GonzalezDelgado, R. M., Pedlar, A., Perez-Fournon, I., Perry, J. J., and Tadhunter, C. N., 1995, A\&A, 302, 58

Winge, C., Axon, D.J., Macchetto, F.D., Capetti, A. and Marconi, A., 1999, ApJ, 519, 134

Yoshida, M. and Ohtani, H., 1993, PASJ, 45, 407 